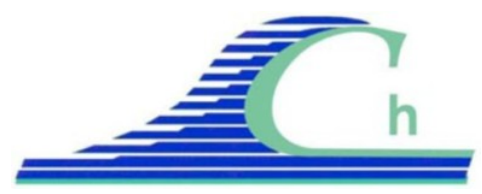

XII ${ }^{\text {ìmes }}$ Journées Nationales Génie Côtier - Génie Civil

Cherbourg, 12-14 juin 2012

DOI:10.5150/jngcgc.2012.018-A @ Editions Paralia CFL

disponible en ligne - http://www.paralia.fr - available online

\title{
Impact des ouvrages portuaires sur la morphodynamique des barres sédimentaires d'avant-côte du Languedoc-Roussillon
}

\author{
Nicolas ALEMAN ${ }^{1}$, Charlotte MICHEL ${ }^{1}$, Nicolas ROBIN ${ }^{1}$, \\ Raphaël CERTAIN ${ }^{1}$, Jean-Paul BARUSSEAU ${ }^{1}$
}

\section{CEFREM, UMR 5110 CNRS - Université de Perpignan, 52 avenue Paul ALDUY, 66860 Perpignan Cedex 9, France. \\ nicolas.aleman@univ-perp.fr}

\section{Résumé :}

Les côtes sableuses du Languedoc-Roussillon sont caractérisées par la présence de systèmes de barres sédimentaires d'avant-côte bien développés. L'implantation massive d'ouvrages portuaires dans les années 70 a transformé les paysages. L'objectif de cette étude est de déterminer l'impact de ces ouvrages sur la morphodynamique des barres. Ce travail se base sur l'analyse de photographies aériennes (IGN) géoréférencées et d'un jeu de données LiDAR sur deux sites d'étude. Il met en avant le rôle des conditions géoenvironnementales préexistantes et des paramètres du port, la relation étroite entre les variations du trait de côte et celles des barres ainsi que l'absence de modification de la typologie.

\section{Mots-clés :}

Dynamique sédimentaire - Côtes sableuses - Ouvrages portuaires - Impact pluridécennal - LiDAR - Golfe du Lion

\section{Introduction}

La mission Racine lancée dans les années 70 a débouché sur l'aménagement touristique du littoral du Languedoc-Roussillon avec la construction de nombreux ports et ouvrages de défense contre la mer. L'avant-côte de ce littoral est caractérisée par la présence de systèmes de barres sableuses bien développés. Ils fournissent une protection naturelle contre les phénomènes d'érosion et de submersion marine.

L’objectif de ce travail est de déterminer l'impact de l'implantation des ports sur ces systèmes de barres d'avant-côte au travers de deux exemples : le port de Canet et le port de Narbonne-Plage. Cette étude se base sur l'analyse d'un jeu de données LiDAR récolté en 2009 (DREAL-LR) et d'un jeu d'anciennes photographies aériennes (IGN) prisent avant et après la construction des ouvrages. Cette méthode diachronique permet ainsi de caractériser la distribution des barres d'avant-côte en milieu naturel (avant la construction des ports) par transparence de l'eau puis de déterminer leur évolution/ajustement vis-à-vis de l'implantation d'un port sur une échelle pluridécennale (30-40 ans). 


\section{Site d'étude}

Le littoral du Languedoc-Roussillon se présente sous la forme d'un linéaire côtier d'environ 200 km constitué principalement de côtes basses sableuses intersectées par quelques caps rocheux. L'avant-côte est constituée par un système de barres pouvant prendre plusieurs configurations morphologiques (linéaires, en croissants....) suivant leur localisation régionale (ALEMAN et al., 2011). Le marnage $(<30 \mathrm{~cm})$ et l'agitation modérées (0,8 m de $\left.\mathrm{Hs}_{\text {moy }}\right)$ néanmoins ponctuées par des tempêtes importantes classent cette région dans un environnement dominé par la houle (HAYES M.O., 1979).

Le port de Canet, construit en 1968, est situé dans la partie sud du LanguedocRoussillon (fig. 1). Son emprise sur la plage immergée est de $370 \mathrm{~m}$. Le trait de côte possède une orientation $\mathrm{N}-\mathrm{S}$ et l'avant-côte présente un système à deux barres festonnées. La dérive littorale principale est dirigée vers le Nord. A $1 \mathrm{~km}$ au Nord du port se présente l'embouchure du fleuve Têt suivi par le port de Sainte Marie-Plage situé sur un ancien débouché du fleuve (fig. 3A).

Le port de Narbonne-Plage, construit en 1980, est situé dans la partie centrale du Languedoc-Roussillon (fig. 1). Son emprise sur la plage immergée est de $337 \mathrm{~m}$. Le trait de côte a une orientation NE-SO et l'avant-côte est marquée par un système à deux barres avec une barre interne festonnée et une barre externe linéaire. La dérive littorale a une direction préférentielle vers le SO. A $940 \mathrm{~m}$ au NE du port apparaît un petit promontoire rocheux, le roc de la Batterie (fig. 3B).

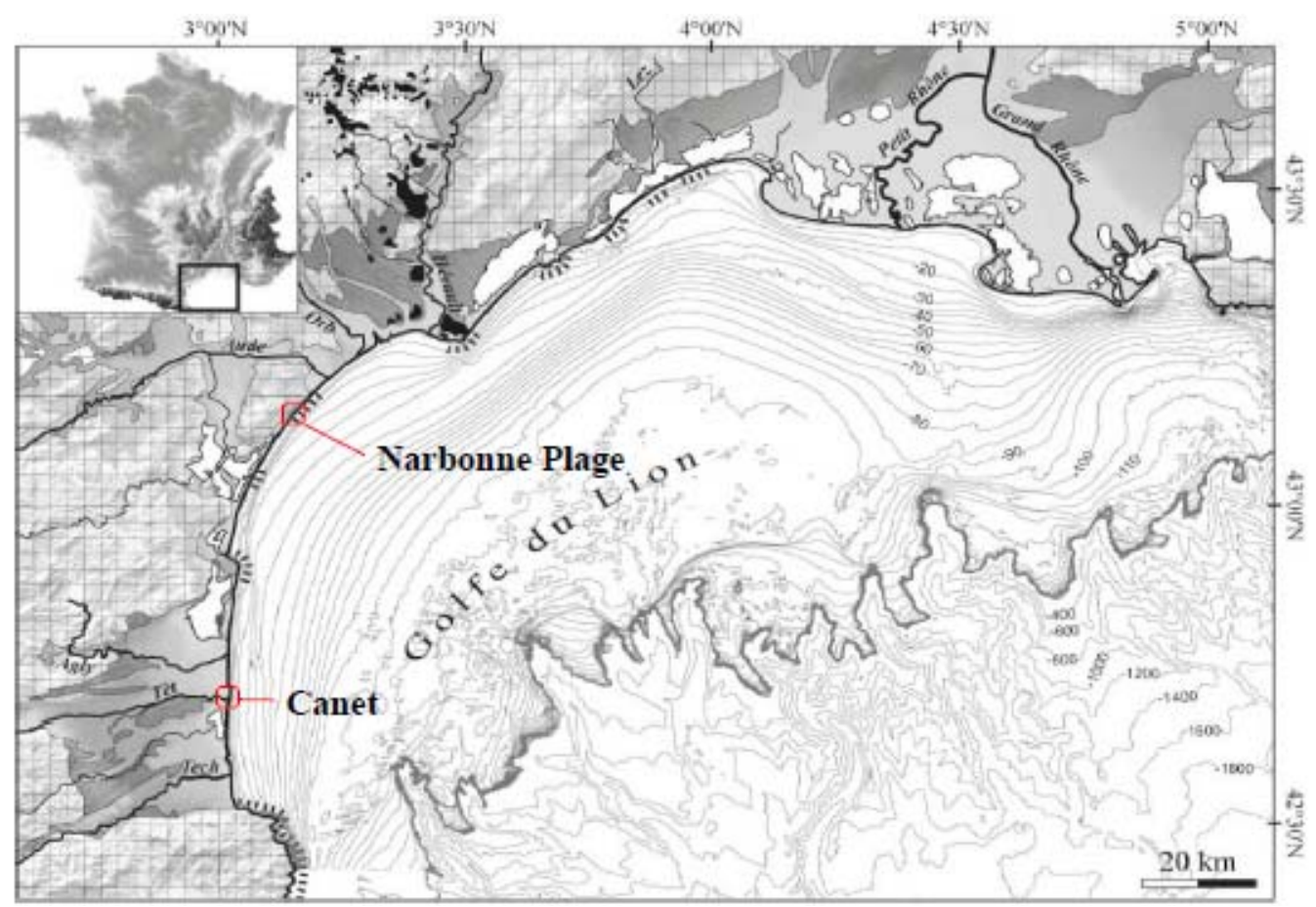

Figure 1. Emplacement des deux ports étudiés au sein du golfe du Lion (carte bathymétrique de l'IFREMER). 


\section{XII ${ }^{\text {èmes }}$ Journées Nationales Génie Côtier - Génie Civil \\ Cherbourg, 12-14 juin 2012}

\section{Méthodologie}

Le jeu de données utilisé est constitué de photographies aériennes de 1962, 1977, 1980, 1989, 1992 et 1995 pour le port de Canet et de 1954, 1986, 1989, 1992 et 1995 pour le port de Narbonne-Plage. Chaque photographie a été géoréferencée à l'aide du logiciel ArcGIS 10. Ces données sont complétées par un MNT issu d'une campagne LiDAR topo-bathymétrique (Capteur LADS MkII de Fugros-Lads) réalisée en 2009 (DREALLR). Les crêtes des systèmes de barres ont été digitalisées pour chaque année sur une distance arbitraire de $2 \mathrm{~km}$ en aval et $2 \mathrm{~km}$ en amont-dérive du port (fig. 2). L'incertitude liée à la donnée brute, au processus de rectification et à la digitalisation est estimée à +/-4 m correspondant à une marge d'erreur classique pour ce type de démarche (BOAK et TURNER, 2005; CROWELL et al., 1991).

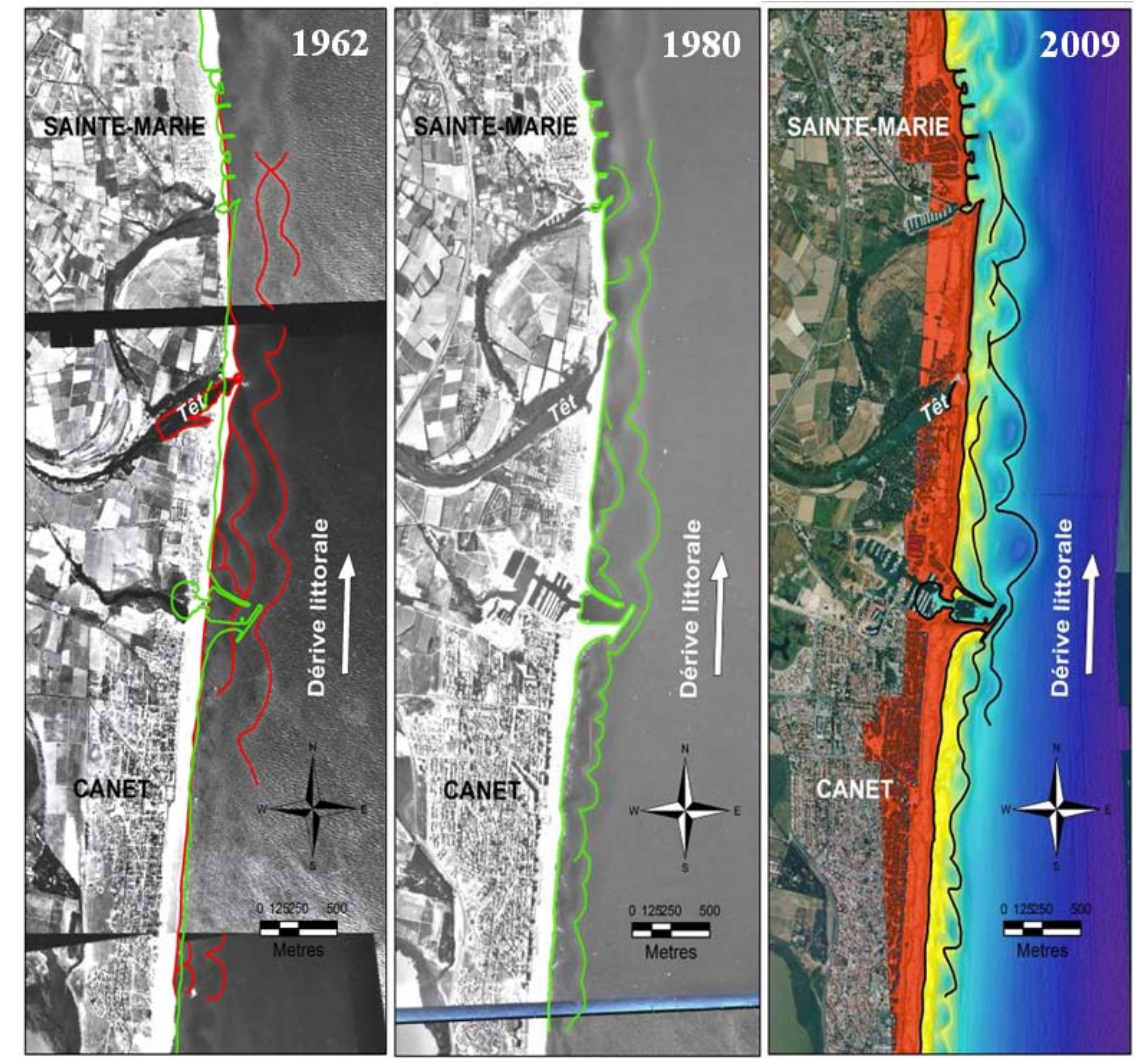

Figure 2. Exemple de photographies aériennes en 1962 (avant construction du port), 1980 et de la donnée LiDAR 2009 à Canet avec la digitalisation des barres d'avantcôte. Pour 1962, la position du trait de côte de 2009 est indiquée (en vert).

\section{Résultats}

La photographie aérienne prise avant la construction des ouvrages portuaires nous informe sur la morphologie des barres sans perturbations anthropiques et sera considérée comme correspondant à l'état initial du système. Sur les deux secteurs étudiés, le contexte géo-environnementale conditionne la morphologie de l'avant-côte. 
En effet, sur le site de Canet (photographie de 1962), le lobe deltaïque créé par l'embouchure de la Têt rompt la barre interne qui vient s'accoler au rivage (fig. 3C). La barre externe passe alors en position de barre interne en aval-dérive et une nouvelle barre externe se crée plus au large. Au niveau de Narbonne Plage (photographie de 1954), le roc de la Batterie joue le rôle d'épis en amont de l'emplacement du futur port. Il engendre un décalage du trait de côte par accumulation en amont-dérive et érosion en aval-dérive (fig. 3D). Ce décalage permet l'apparition d'une troisième barre près du rivage en aval-dérive, directement enracinée sur le roc. Cette barre est festonnée certainement en raison de la position plus éloignée de la barre interne qui atténue moins l'énergie de la houle.
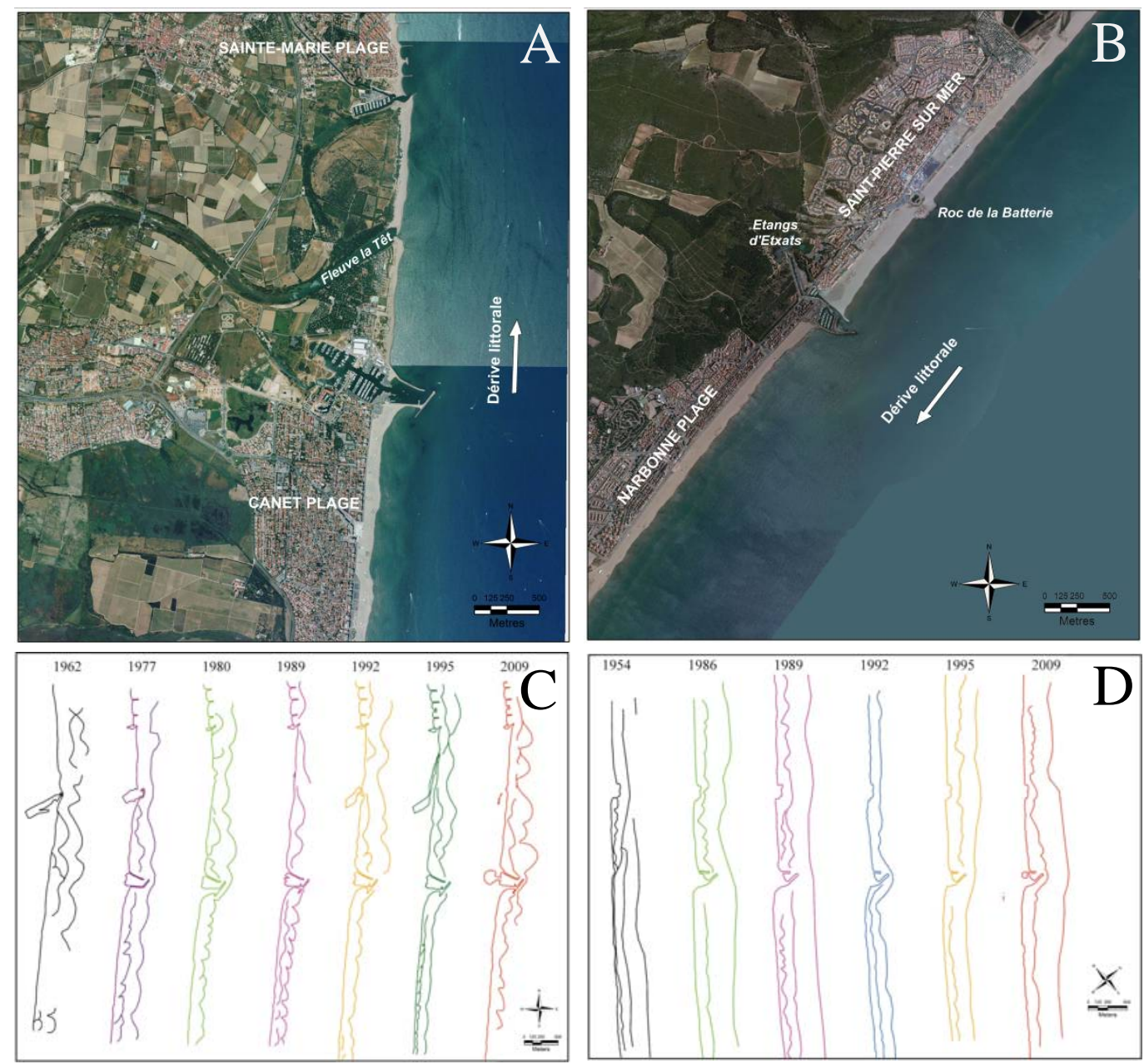

Figure 3. Photographies aériennes du port de Canet (A) et du port de Narbonne Plage

(B) et les systèmes de barres digitalisées pour 7 années à Canet $(C)$ et 6 années à Narbonne Plage (D).

Dès la création du port Canet en 1968, le trait de côte subit une avancée (jusqu'à $1 \mathrm{~m} / \mathrm{an}$ sur l'ensemble de la période) en amont-dérive (fig. 4). Néanmoins, cette tendance 


\section{XII ${ }^{\text {èmes }}$ Journées Nationales Génie Côtier - Génie Civil \\ Cherbourg, 12-14 juin 2012}

diminue en se rapprochant de l'ouvrage jusqu'aux derniers $500 \mathrm{~m}$ où un recul est observée (0,2 m/an) (fig. 4). En aval-dérive, l'évolution de l'avant-côte apparait plus complexe. Dès la construction du port, le littoral subit un recul. La perturbation du transit sédimentaire ainsi créée induit la destruction progressive du lobe deltaïque de la Têt (recul de $115 \mathrm{~m}$ sur la période étudiée (environ $-2.4 \mathrm{~m} / \mathrm{an}$ )) où les sédiments sont redéposés plus en aval au voisinage du port de Sainte-Marie (avancée de $0.8 \mathrm{~m} / \mathrm{an}$ sur l’ensemble de la période).

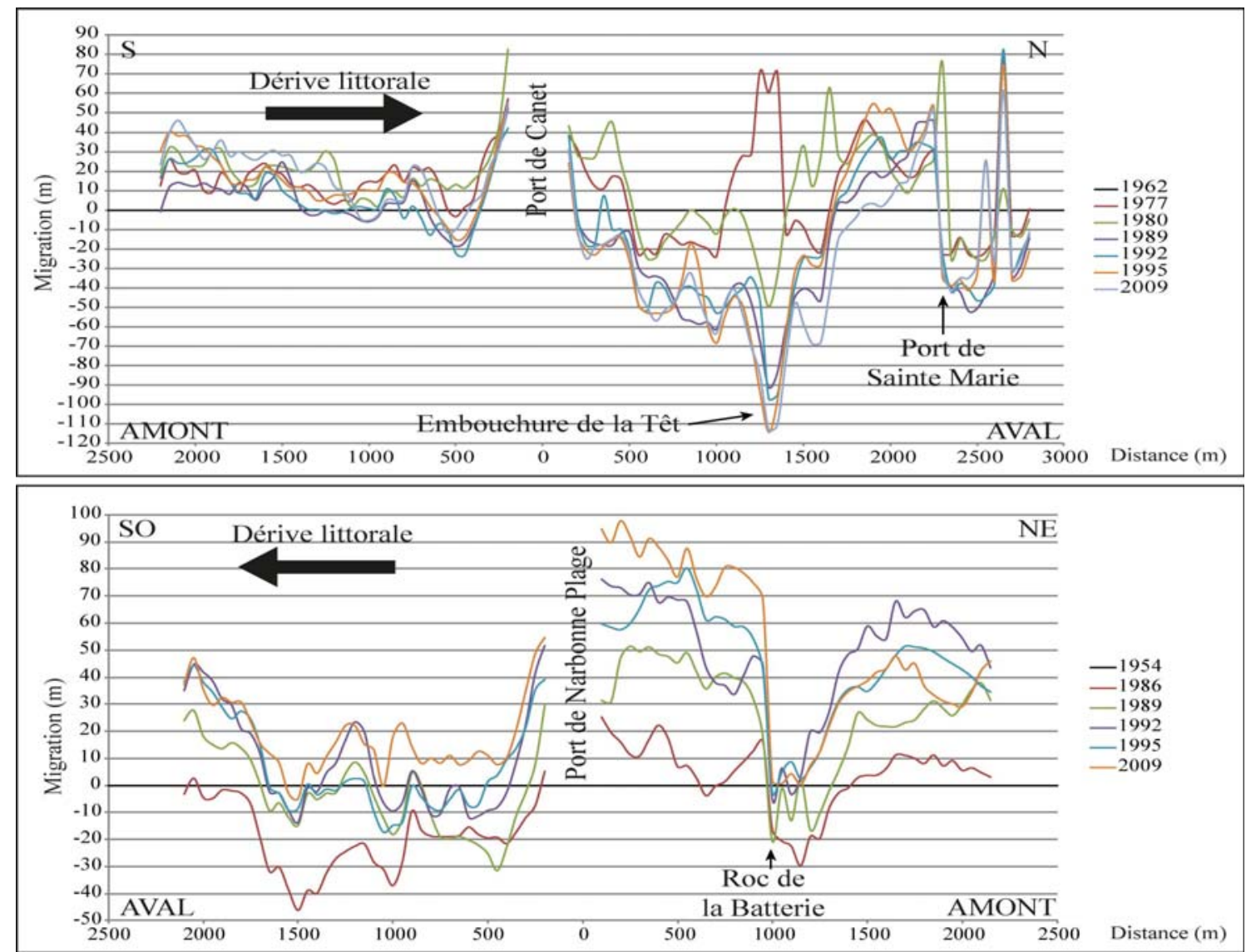

Figure 4. Migration du trait de côte au voisinage des ports de Canet et Narbonne. La valeur d'avancée du trait de côte de chaque année est donnée par rapport à 1954.

La construction du port de Canet en 1968 n'engendre pas de bouleversements sur la typologie des barres qui restent festonnées. Néanmoins, des changements apparaissent sur leur continuité. Le premier est la rupture de la barre interne dès la construction de l'ouvrage (fig. 3C). La barre externe arrive à franchir cet obstacle mais sa rupture est possible comme l'atteste les années 1989, 1992 et 1995. La barre interne en aval-dérive était initialement rompue par le delta de la Têt. Le recul progressif du lobe lui permet finalement de retrouver une continuité à partir de 1995 (fig. 3C). La distance de la barre 
externe au trait de côte ne varie pas plus d'une dizaine de mètres en amont comme en aval et la position des barres semblent suivre plus ou moins l'évolution du trait de côte. La construction du port de Narbonne Plage en 1980 engendre immédiatement une importante avancée du trait de côte en amont-dérive qui perdure sur l'ensemble des levés (jusqu'à 3,4 m/an sur une période de 29 ans) (fig. 4). Bien que le l'intervalle de temps entre les deux photos soit importante (1954-1980), l'expertise des évolutions diachroniques laisse supposer la part importante jouée par l'ouvrage dans cette tendance. Ainsi, l’irrégularité du littoral formée par le roc de la Batterie sur la période antérieure s'homogénéise suite à la construction du port qui perturbe le transit sédimentaire (fig.4). En aval-dérive, le trait de côte est en érosion entre 1954 et 1986. Cette évolution est supposée être engendrée par la construction du port en 1980 (recul de $30 \mathrm{~m}$ en moyenne). Entre 1986 et 2009, la tendance s'inverse (0,8 m en moyenne) et le trait de côte de 2009 se retrouve en position avancée par rapport au trait de côte de 1954.

La typologie du système de barre ne semble pas être affectée par la présence de l'ouvrage où leur configuration reste inchangée. Lors de sa construction, les digues du port rompent la barre interne. Cependant, le système de barres migre vers le large. A l'inverse du site de Canet, ce recul général du système de barres permet en 2009 à la barre interne de franchir de nouveau le port (fig. 3D).

\section{Interprétation et discussion}

Il est avéré que la construction d'un ouvrage transversal induit des modifications sur l'évolution du trait de côte d'autant plus si il est de dimension importante comme c'est le cas des ouvrages portuaires (e.g. SAMAT, 2007 ; RITPHRING et TANAKA, 2011 ; KUDALE, 2010). La perturbation du transit sédimentaire provoque généralement une avancée du trait de côte en amont-dérive et un recul en aval-dérive. Les deux sites d'étude n'échappent pas à cette tendance avec cependant des comportements différents. Narbonne plage présente une avancée du trait de côte en amont-dérive très supérieure à celle de Canet et sur un linéaire plus important. A l'inverse, l'aval-dérive se caractérise par un recul plus important sur le site de Canet. Par contre, il est à noter qu'une avancée significative est observée contre l'ouvrage pendant les premières dizaines de mètres. Cette tendance perdure tant que le système perturbe fortement le transit sédimentaire. Lorsque l'avancée amont-dérive devient importante, le transit peut de nouveau alimenter, dans une certaine mesure, le système aval et induire une avancée de celui-ci. Ce cas est parfaitement illustré sur le site de Narbonne Plage où à partir des années 1995, un transit probablement important franchit le port (souligné par le franchissement ininterrompu de la barre interne) et la position du trait de côte en 2009 est la plus avancée jamais observée. Sur le site de Canet, l'avancée en amont-dérive est trop faible pour observer un tel comportement. 


\section{XII ${ }^{\text {èmes }}$ Journées Nationales Génie Côtier - Génie Civil \\ Cherbourg, 12-14 juin 2012}

L'avancée forcée du trait de côte par les structures anthropiques a une conséquence proche de l'ouvrage mais également sur un linéaire plus éloigné tant en amont qu'en aval-dérive. Le littoral aval du site de Canet se caractérise par un lobe deltaïque à 1300 $\mathrm{m}$ du port (embouchure de la Têt). Ce lobe s'est crée par la succession des événements de crues, dont la dernière majeure s'est produite en 1940, et présente une érosion depuis le cliché de 1980. Cette tendance au recul est à mettre en lien avec la chute des apports fluviaux dut à la construction d'un important barrage en amont en 1978 (BOURRIN, 2007). Ce processus à été démontré sur plusieurs fleuves côtiers méditerranéens (JABALOY-SÁNCHEZ et al., 2010). Néanmoins, la perturbation ou le blocage du transit sédimentaire par le port de Canet est un élément supplémentaire néfaste pour cette partie de côte renforçant ou accélérant l'érosion de ce lobe deltaïque. Autre exemple, en amont du port de Narbonne plage, la présence du Roc de la Batterie à 940 $m$ des jetées induisait un décrochement du trait de côte entre celui-ci et le futur lieu d'implantation du port. Sa construction et sa conséquence direct sur l'avancée du trait de côte de cette partie de littoral rend maintenant négligeable l'effet de ce promontoire rocheux. Ainsi, cette étude montre que l'impact des ports peut avoir des conséquences positives ou négatives sur l'évolution du trait de côte jusqu'à une distance d'éloignement d'au moins de $1300 \mathrm{~m}$. Les travaux de Michel et al (2011) montrent que cette distance est en relation direct avec la dimension des ouvrages.

Peu de travaux se sont penchés sur l'impact des ports sur l'évolution des barres d'avantcôte. Cette étude révèle que leur typologie reste inchangée. Elle est contrôlée par le contexte géo-environnementale de la zone d'étude et les changements locaux induis par la construction du port sur le disponible sédimentaire ou l'hydrodynamisme sont insuffisant pour les faire changer significativement de morphologie. Par contre, l'implantation des ports a un impact sur la continuité des barres. Dans les deux sites d'étude, la barre interne est rompue. Cet impact peut s'étendre également à la barre externe (cas de Canet). Cependant, l'avancée du trait de côte amont et l'accrétion probable de cette avant-côte permet le recul des barres et le possible franchissement de la barre interne (cas de Narbonne plage). Cette observation a déjà été reportée par différents auteurs (SZMYTKIEWICZ et al., 2000 ; MANGOR et al., 2010). Ainsi, il est montré à travers ces deux exemples, un déplacement de l'ensemble du système « trait de côte-plage-barres » vers le large en amont-dérive pouvant avoir des répercussions positives sur des échelles pluri-décennales sur l'évolution du système aval (continuité des barres, rétablissement des apports sédimentaires, ...).

\section{Conclusion}

L'étude diachronique de photographies aériennes géoréférencées constitue un outil performant de détermination de l'impact d'un ouvrage portuaire sur l'évolution du trait de côte et la morphologie des barres sédimentaires d'avant-côte. Ainsi, ce travail a permis de mettre en évidence : (1) la construction des ouvrages portuaires engendre une 
accumulation sédimentaire en amont-transit entrainant une avancée du trait de côte et une érosion en aval-transit avec un recul du trait de côte. Ce phénomène est modéré par le contrôle géo-environnemental local mais peut influencer l'évolution naturelle du trait de côte sur au moins $1300 \mathrm{~m}$; (2) la migration des barres d'avant-côte est liée à la position du trait de côte. Une barre initialement rompue par les jetées de l'ouvrage peut parvenir à le franchir avec l'avancée du trait de côte et rétablir ainsi une partie du transit sédimentaire. Les paramètres d'ingénierie du port, dont principalement l'emprise et la forme des jetées, influence ce mécanisme et (3) la typologie générale des barres n’est pas affectée.

Cependant, l'utilisation de cette méthodologie peut induire des biais dans les interprétations. La qualité des photographies aériennes peut gêner l'identification de la position des barres notamment pour une étude micro-échelle comme l'évolution de leurs paramètres morphométriques, ce qui n'est pas l'objectif de ce travail qui se situe sur une échelle globale. Les séries temporelles irrégulières peuvent également rendre difficile la différentiation entre les mécanismes se produisant à courte et longue échelle de temps. Enfin, le travail sur photographies aériennes permet de travailler uniquement en système planimétrique occultant les informations en trois dimensions tels que l'évolution des pentes et du budget sédimentaire de l'avant côte, ou les caractéristiques morphométriques des barres. Ainsi, la confrontation des données LiDAR 3D de 2009 et d'un nouveau vol réalisé en 2011 permettront d'approfondir ces biais. Ce travail, en cours, s'étendra sur un panel de ports plus important afin de prendre en considération différents contextes géologiques présents sur le littoral du Golfe du Lion.

\section{Remerciements}

Les auteurs remercient la DREAL-LR pour la mise à disposition des données LiDAR topo-bathymétriques et le financement de la thèse de N. ALEMAN.

\section{Références bibliographiques}

ALEMAN N., ROBIN N., CERTAIN R., VANROYE C., BARUSSEAU J.P., BOUCHETTE F. (2011). Typology of nearshore bars in the Gulf of Lions (France) using LiDAR technology. Journal of Coastal Research, SI64, pp 721-725.

BOAK E.H., TURNER I.L. (2005). Shoreline definition and detection: a review. Journal of Coastal Research, Vol. 21(4), pp 688-704. doi:10.2112/03-0071.1

BOURRIN F. (2007). Variabilité et devenir des apports sédimentaires par les fleuves côtiers : cas du système Têt - littoral roussillonnais dans le golfe du Lion. Thèse de doctorat, Université de Perpignan, 305 p.

CROWELL M., LEATHERMAN S.P., BUCKLEY M.K. (1991). Historical shoreline change: error analysis and mapping accuracy. Journal of Coastal Research, Vol. 7(3), pp 839-852. 
HAYES M.O. (1979). Barrier island morphology as a function of tidal and wave regime, in Leatherman, S.P., ed., Barrier islands from the Gulf of St. Lawrence to the Gulf of Mexico: New York, Academic Press, pp 211-236.

JABALOY-SÁNCHEZ A., LOBO F.J., AZOR A., BARCENAS P., FERNADESSALAS L.M., DIAZ DEL RIO V., PEREZ-PEÑA J.V. (2010). Human-driven coastline changes in the Adra river deltaic system, southeast Spain. Geomorphology, Vol. 119, pp 9-22. doi:10.1016/j.geomorph.2010.02.004

KUDALE M.D. (2010). Impact of port development on the coastline and the need for protection. Indian Journal of Geo-Marine Sciences, Vol. 39(4), pp 597-604.

MICHEL C., ALEMAN N., ROBIN N., CERTAIN R., GUERINEL B., VANROYE C., BARUSSEAU J.-P., BOUCHETTE F. (2011). Modification des configurations de barres d'avant-côte au voisinage des ports de plaisance : exemple du Languedoc-

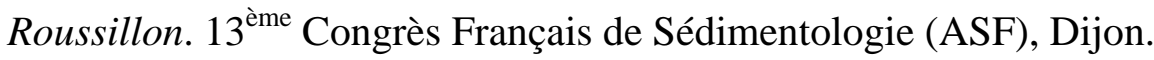

MANGOR K., BROKER I., DEIGAARD R., GRUNNET N. (2010). Bypass harbours at littoral transport coasts. PIANC MMX Congress, Liverpool, UK, pp 1-18.

RITPHRING S., TANAKA H. (2011). Morphology variability in the vicinity of coastal structures. Proceedings of $32{ }^{\text {nd }}$ Conference on Coastal Engineering, Shanghai, China

SAMAT O. (2007). Efficacité et impact des ouvrages en enrochement sur les plages microtidales. Le cas du Languedoc et du delta du Rhône. Thèse de doctorat, Université Aix-Marseille I, 370 p.

SZMYTKIEWICZ M., BIEGOWSKI J., KACZMAREK L.M., OKROJ T., OSTROWSKI R., PRUSZAK Z., ROZYNSKY G., SKAJA M. (2000). Coastline changes nearby harbor structures: comparative analysis of one-line models versus field data. Coastal Engineering, Vol. 40, pp 119-139. doi:10.1016/S0378-3839(00)00008-9 
Thème 2 - Dynamique sédimentaire 